significantly higher in the clinically indicated CXR group (76.9\%) rather than the routine CXR group $(2.7 \%)$ ( $\mathrm{p}=$ $<0.001)$. The therapeutic efficacy was $53.8 \%$ for clinically indicated CXRs, whereas the routine CXRs had a therapeutic efficacy of $1.3 \%$ ( $p=<0.001$ ). There was a significant association between CXR findings and whether the CXR requested was routine or not $(\mathrm{X} 2(1)=70.07, \mathrm{p}<0.001)$ and also management changes (X2 $(1)=45.43, \mathrm{p}<0.001)$.

Conclusion Routine CXR in patient admitted with STEMI are often unnecessary and may add very little clinical value. On the other hand, selective post-procedural CXRs have a significantly higher diagnostic and therapeutic yield. Our study questions the rationale behind routine CXRs in the care of patients with STEMIs.

Conflict of Interest None to declare

\section{THE IMPACT OF FRAILTY ON THE OUTCOMES OF PERCUTANEOUS CORONARY INTERVENTION (PCI) IN THE SETTING OF NON-ST ELEVATION MYOCARDIAL INFARCTION}

'Zhihong Yao, 'Simon Thackray, ${ }^{2}$ Anam Khurram. 'Scunthorpe General Hospital, Scunthorpe, UK; ${ }^{2}$ Hull and York Medical School

\subsection{6/heartjnl-2021-BCS.53}

Introduction With the increase in longevity, older and frailer patients represent a growing cohort for percutaneous coronary intervention (PCI). Frail patients represent a high risk subset and have been shown to predict adverse outcomes more reliably than age. Evidence suggests that PCI may be useful in those who are at high risk for recurrent events. However, frail patients are under-represented in clinical trials with only "fitter" elderly people being included and therefore not being representative of the real-world elderly population. The decision making on intervention regarding elderly or frail patients presenting with non-ST elevation myocardial infarction (NSTEMI) is therefore difficult. The aim of the study is to evaluate the outcomes of NSTEMI PCI with age and frailty independently.

Method Frailty scoring was done retrospectively using the CSHA Frailty Scale for patients presenting to the trust from Jan'16 to Oct'16 \& undergoing PCI for NSTEMI. They were stratified according to age $(<80 \& \geq 80$ yrs) and frailty - not frail (score of 1-3) \& frail (4-6). Outcomes were length of hospital stay, complications and all-cause mortality at 1-year. The association between age and each outcome was examined using Spearman's Rank Correlation; this was also done for frailty.

Result Frailty was assessed in 106 patients with a mean age of $73.5 \pm 12$ years, $65 \%$ were males. 73 (69\%) were not frail and $33(31 \%)$ were vulnerable/frail. The RS between age and frailty was 0.41 indicating moderate correlation between the two variables, $20 / 33(61 \%)$ of the frail group were under 80 . Older and frail group both had higher rates of mortality, contrast nephropathy and longer hospital stay. When the entire cohort was analysed using Spearman's, frailty correlated more strongly with mortality $(\mathrm{r}=0.41 ; \mathrm{p}=0.01)$ and length of stay $(\mathrm{r}=0.3 ; \mathrm{p}=0.04)$ than age however, readmission correlated more strongly with age $(r=0.3 ; p=0.02)$. The association between both age and frailty with nephropathy \& bleeding was not statistically significant nor was the relationship between age and length of stay $(p>0.05)$. Readmission was higher in the frail group but not in the elderly due to noncoronary issues in most.

Conclusion Frail patients, even when younger, have similar outcomes to very old patients, particular with respect to death, kidney damage and subsequent long hospital stay in NSTEMI patients. Additionally, frail score appears to be better predictor for hospital readmission rate compared to age alone. Even in those patient under 80 , frailty score appears to predict these with poorer outcomes, suggesting decision based purely on age are invalid.

Conflict of Interest None

\section{CHANGING INCIDENCE AND PROCEDURE COMPLEXITY OF CORONARY ARTERY PERFORATIONS. - A 10-YEAR DATASET}

${ }^{1}$ Hamza Umar, ${ }^{1}$ Ashwin Roy, Mohammed Oshieba, ${ }^{1}$ Peter Ludman, ${ }^{1} J o n a t h a n$ Townend ${ }^{2}$ M. Adnan Nadir, ${ }^{3}$ SN Doshi, 'S Sudhakar George, ${ }^{1}$ Alex Zaphiriou, ${ }^{2}$ Sohail Q Khan. 'Queen Elizabeth Hospital, University Hospital Birmingham, Birmingham, UK; ${ }^{2}$ Queen Elizabeth Hospital Birmingham; ${ }^{3}$ Dept of Cardiology, Queen Elizabeth Hospital, Birmingham, UK

\subsection{6/heartjnl-2021-BCS.54}

Background Coronary artery perforation (CAP) is a life-threatening complication of percutaneous coronary intervention (PCI). This study aims to identify CAP predictors using contemporary data.

Methods Retrospective cohort study on all PCIs performed at the Queen Elizabeth Hospital, Birmingham between January 2010 and October 2020. Patient demographics, comorbidities, modified Ellis perforation class and perforation treatments were collected. The CAP population was split into two cohorts (1 and 2), representing the first and second 5-year period in the decade.

Results During a 10-year period, 9,504 PCI procedures were performed with CAP occurring in 66 cases. According to the Ellis criteria, $4.5 \%$ of CAPs were type I, $13.6 \%$ type II, $45,5 \%$ type III, and $34.8 \%$ type V. Definitive management included balloon inflation only in $27 \%$, covered stents $20 \%$,

\begin{tabular}{|c|c|c|c|}
\hline & $\begin{array}{l}\text { Cohort 1: }(n= \\
23)\end{array}$ & $\begin{array}{l}\text { Cohort 2: }(n= \\
43)\end{array}$ & $\begin{array}{l}\text { Total PCI }(n= \\
9504)\end{array}$ \\
\hline Mean Age and Standard & $69.3+/-11.3$ & $70.6+/-13.2$ & $64.6+/-12.4$ \\
\hline \multicolumn{4}{|l|}{ Deviation } \\
\hline Male \% & 52.2 & 69.8 & 72.9 \\
\hline Incidence \% & 0.55 & 0.81 & 0.69 \\
\hline Hypertension \% & 69.6 & 65.1 & 59.5 \\
\hline Hypercholesterolaemia \% & 69.6 & 44.2 & 49.7 \\
\hline Chronic Kidney Disease (CKD) \% & 0 & 4.65 & 1.90 \\
\hline Diabetes \% & 13.0 & 28.0 & 26.9 \\
\hline Acute Coronary Syndrome (ACS) & 87.0 & 65.1 & 68.6 \\
\hline \multicolumn{4}{|l|}{$\%$} \\
\hline Сто \% & 8.70 & 25.6 & 6.06 \\
\hline Use of GP IIb/Illa Inhibitors \% & 30.4 & 16.3 & 30.0 \\
\hline Intravascular Ultrasound (IVUS) & 13.0 & 18.6 & 8.86 \\
\hline \multicolumn{4}{|l|}{$\%$} \\
\hline Rotablation \% & 4.35 & 9.30 & 3.23 \\
\hline Tamponade \% & 8.70 & 25.6 & 0.24 \\
\hline Hydrophilic wires \% & 34.8 & 34.9 & 15.0 \\
\hline Mortality \% & 8.70 & 16.3 & 2.58 \\
\hline
\end{tabular}


conservative 20\%, balloon inflation with heparin reversal $12 \%$, coronary coils $12 \%$, heparin reversal $4.5 \%$, emergency surgery $3 \%$, coil and covered stent $1.5 \%$. Overall mortality within the total perforation cohort was 14\%. Emergency pericardiocentesis was required in 13 patients; in this group mortality was $46 \%$. The difference in trends is illustrated in the table 1 .

Conclusion The data demonstrates increased incidence of CAP and mortality in the second half of the decade. This may be explained by an increase in CTO, rotablation and IVUS use (surrogates for complex PCI) and an older population with more comorbidities. Cardiac tamponade was associated with a higher mortality in those with CAP.

Conflict of Interest none

\section{LONG TERM CLINICAL OUTCOMES FROM USE OF SIROLIMUS COATED BALLOON IN CORONARY INTERVENTION; DATA FROM A REAL-WORLD POPULATION}

Bhagya Harindi Loku Waduge, Harkaran Kalkat, Ameenathul Mazaya Fawzy, Abdullah Saif, Sampath Athukorala, Gurbir Bhatia, Nitin Kumar, Bethan Freestone, Jerome Ment, Kaeng Lee, Michael Pitt, George Pulikal, Sandeep Basavarajaiah Sandeep Basavarajaiah. Birmingham Heartlands Hospital, Birmingham, UK

\subsection{6/heartjnl-2021-BCS.55}

Background The use of drug coated balloons (DCBs) in coronary intervention is escalating. There are two drugs of choice for coating either DCBs or drug eluting stents: Paclitaxel or Sirolimus. Most available DCBs are coated with Paclitaxcel, due to pre-existing, extensive data that support good clinical outcomes. With drug eluting stents both the literature and hence clinical practice favour Sirolimus over Paclitaxcel. This is due the cytostatic properties and wide therapeutic window of Sirolimus. However, there is very limited data on Sirolimus coated balloons (SCBs). We report a long-term follow-up with this relatively new technology from our centre.

Methods A retrospective analysis was conducted on all patients treated with an SCB between March 2018 and October 2020. Follow-up was achieved with clinic visits, telephone calls and admission records. The outcomes measured include cardiac death, target-vessel myocardial-infarction, target lesion revascularisation and MACE (combination of cardiac death, targetvessel MI and TLR).

Results 533 patients (690-lesions) with a mean age of 65.4 (range; 37-90) were treated with an SCB. 79\% ( $n=419)$ were male, $314(59 \%)$ were in the setting of acute coronary syndrome, 40\% $(n=211)$ had diabetes and 60\% $(n=414)$ had DCB in de-novo lesions. Small vessels accounted for $59 \%$ of cases $(n=406)$. Pre-dilatation was performed in 97\% $(n=670)$ of cases. Bailout stenting (with a drug eluting stent) was required in $6.5 \%$ lesions $(n=45)$, of which 11 were due to dissections and 34 were due to $>50 \%$ recoil following DCB use. The mean diameter and length of DCBs were $2.8 \mathrm{~mm}$ and $26.3 \mathrm{~mm}$ respectively. During a median follow-up of 572 days (IQR: 381 - 868); cardiac death occurred in 15 patients (3\%). Target vessel MI was in $4 \% ; n=21$, TLR per lesion and per patient were $10 \%(\mathrm{n}=72$ and $\mathrm{n}=55$ respectively). The overall MACE rate was $12 \%$. There were no documented cases of acute vessel closure.

Conclusions The results from long term follow-up with this relatively new technology DCB are encouraging with low rates of hard endpoints and acceptable rates of TLR and MACE despite complex group of patients (59\% ACS and 40\% diabetics) and lesion subsets (40\% restenotic lesions and 59\% small vessels). This suggests that SCBs can be used in both restenotic and de novo small vessel lesions with acceptable clinical outcomes. However, in order to further inform clinical practice, more longer-term data on SCBs compared with Paclitaxel coated balloons is needed.

Conflict of Interest None to declare

\section{USE OF SIROLIMUS COATED BALLOON IN DE NOVO SMALL VESSEL CORONARY LESIONS; LONG-TERM FOLLOW-UP FROM A SINGLE CENTRE REGISTRY}

Bhagya Harindi Loku Waduge, Harkaran Kalkat, Ameenathul Mazaya Fawzy, Abdullah Saif, Sampath Athukorala, Gurbir Bhatia, Nitin Kumar, Bethan Freestone, Jerome Ment, Kaeng Lee, Michael Pitt, George Pulikal, Sandeep Basavarajaiah Sandeep Basavarajaiah. Birmingham Heartlands Hospital, Birmingham, UK

\subsection{6/heartjnl-2021-BCS.56}

Background Drug coated balloons (DCBs) in Europe are mainly used in restenotic lesions as endorsed by the European Society of Cardiology, with a class IA recommendation. However, some of the recent data suggest, it can also be considered in a subset of denovo lesions, especially in small vessels. Most DCBs used are coated with Paclitaxcel. There is no data on the efficacy of Sirolimus in DCBs, the drug of choice in drug eluting stents. In this study, we report outcomes from the use of a Sirolimus coated balloon (SCB) in de novo small-vessel coronary lesions, from a single high yield centre.

Methods A retrospective analysis was conducted on all patients treated with an SCB between March 2018 and October 2020. Follow-up was achieved with clinic visits, telephone calls and admission records. The outcomes measured include cardiac death, target-vessel myocardial-infarction, target lesion revascularisation and MACE (combination of cardiac death, targetvessel MI and TLR).

Results During the study period, 279-patients (with 332lesions) with de novo lesions were treated with an SCB. The mean age of patients was $65 \pm 12$ years, 219 (79\%) were male, 36\% $(n=100)$ had diabetes, 16\% $(n=45)$ had chronic kidney disease and $61 \%$ were in the setting of acute coronary syndrome $(n=169)$. Predilatation was performed in 96\% (320lesions). Bailout stenting (with DES) was required in $5 \%$ lesions $(n=18)$ and of which 16 were due to dissections and 2 were due to recoil $>30 \%$ following DCB use. The mean diameter and length of DCBs were $2.35 \mathrm{~mm}$ and $26 \mathrm{~mm}$ respectively. During a median follow-up of 584-days (19months) cardiac death was reported in 8 patients (3\%). Target vessel MI was in 3\% $(n=9)$, TLR per lesion was $8 \%(n=26)$ and the MACE rate was $11 \%(n=31)$. There were no documented cases of acute vessel closure.

Conclusion The long-term outcome from the first ever study on sirolimus eluting balloon in de novo small vessel lesions appears promising with low rates of hard endpoints, and acceptable repeat rates of TLR despite a complex group of patients (50\% ACS, 36\% diabetics and 19\% CKD) and lesion subsets (small vessel and diffuse disease). Implanting stents in these subsets renders them vulnerable to restenosis, making it difficult to treat, making treatment challenging and resulting in high rates of recurrence.

Conflict of Interest None to declare 